\title{
ZS Research Square \\ Sn-doped CdS: A New Intermediate Band Material for Solar Cells
}

Jianbo Yin ( $\nabla$ jianbery@163.com )

Lanzhou University of Technology

Xiaobin Yan

Lanzhou University of Technology

Min Zhu

Lanzhou University of Technology

\section{Research Article}

Keywords: cadmium sulfide, intermediate band, doping, tin

Posted Date: November 23rd, 2021

DOI: https://doi.org/10.21203/rs.3.rs-884668/v1

License: (1) This work is licensed under a Creative Commons Attribution 4.0 International License.

Read Full License 


\section{Abstract}

In this paper, the electronic structure and optical properties of CdS doped by Sn with different concentrations were investigated by first principles. The calculation results of electronic structure show that the doping of Sn can produce a deep impurity level band in the band structure of CdS. The calculation results of optical property show that Sn doping can increase the light absorption coefficient and conductivity of CdS. The overall calculation results show that $\mathrm{Sn}$ doping can produce stable intermediate band structure and significantly improve the optical property of CdS.

\section{Introduction}

At present, the rapid development of industrialization leads to the global energy shortage, and a large number of toxic and harmful chemical pollutants are discharged into the environment. Semiconductor optoelectronic material is one of the solar energy fuels to solve the global energy shortage and environmental pollution. On the one hand, semiconductor optoelectronic technology can utilize solar energy to produce electric energy [1-5], such as obtaining current through photovoltaic materials [6], producing fluorescence and phosphorescence by photoluminescent materials $[7,8]$; on the other hand, photovoltaic technology can replace various toxic and harmful chemical fossil energy to purify the environment [9-11]. CdS is a direct band gap semiconductor [12-15], which has excellent photoelectric performance, such as high photoelectric conversion efficiency, low cost, long service life, rich lightemitting color and so on [16-18]. However, due to its large band gap (2.42 ev) [19], CdS can only absorb a little energy from the visible light and can not make full use of the visible light. Therefore, it is necessary to adjust the band structure of CdS to expand its visible light response range. Scholars have used a variety of means to expand its spectral range, such as composite[20], sensitization[21], nanosizing[22] and doping[23]. Among those methods, doping is the simplest method with the lowest cost. If a deep impurity level intermediate band structure (shown as Fig. 1) can produce in the band structure of CdS by doping, which is equivalent to inserting a transit station of photon absorption, the idea of doping will be changed. It can be equivalent to the parallel connection of three different band gap semiconductors as shown in Fig. 1, which can not only greatly increase the spectral absorption range of CdS, but also keep its band gap width unchanged, making the theoretical limit photoelectric conversion as high as 63\% [24].

In recent years, using intermediate band to improve the absorption of photons with energy lower than the band gap has been developed continuously. For example, the prediction results of Xue et al. shows that $\mathrm{Sb}$ doped $\mathrm{SnS}_{2}$ can produce intermediate band structure [25]; Wlfa et al. prepared Ti doped $\mathrm{CuGaS}_{2}$ intermediate band material and obtained excellent optoelectronic performance [26]. Tanaka et al. studied $\mathrm{Cl}$-doping effect in $\mathrm{ZnTe}_{1-\mathrm{x}} \mathrm{O}_{\mathrm{x}}$ highly mismatched alloys for intermediate band solar cells and the results showed that the doping of $\mathrm{Cl}$ could significantly change the optoelectronic performance of $\mathrm{ZnTe}_{1-\mathrm{x}} \mathrm{O}_{\mathrm{x}}$ [27]. Therefore, the study results show that the intermediate band structure can greatly improve the photoelectric properties of materials. However, the research on CdS intermediate band material is rarely reported. Based on this, the intermediate band structure and optical property of Sn doped CdS are discussed, the purpose of which is to give suggestion of experiments researchers. 


\section{Calculation Model And Method}

First principles based on density functional theory were adopted to study Sn doped CdS intermediate band material, and all the calculations were performed by MS (Material Studio). The local-density approximation (LDA) is used as the exchange correlation function. In order to obtain the accurate electronic structure, the $U$ value provided through a series of tests, the $L D A+U\left(U_{C d}=U_{S}=5.0 \mathrm{eV}\right)$ method is used to overcome the shortcomings of LDA. The adopted supercell of CdS is $2 \times 2 \times 2$ which contains 64 atoms, including $32 \mathrm{Cadmium}$ atoms and 32 sulfur atoms. The energy cutoff was set for $400 \mathrm{eV}$, and the grid of point $K$ was set for $4 \times 4 \times 4$, respectively. The adopted supercell of CdS is cubic phase structure, which has a space group of F43 $\mathrm{m}(216)$ and a lattice constant of $a=b=c=0.583 \mathrm{~nm}$. The valence electrons involved in the calculation are $C d: 4 d^{1} 5 s^{2}, s: 3 s^{2} 3 p^{4}, S n: 5 s^{2} 5 p^{2}$, respectively.

\section{Results And Discussion}

In order to evaluate the accuracy of the method, the supercell optimization calculations were carried out. Firstly, CdS primitive cell was optimized, and the lattice constant was $a=b=c=0.5832 \mathrm{~nm}$ after optimization, which I $\mathrm{s}$ very consistent with the experimental data of $a=b=c=0.5818 \mathrm{~nm}$ [28], and ensures the reliability of the method. Therefore, the same conditions are used to calculate the electron structure and optical property of Sn doped CdS.

In order to determine the stability of the Sn doped CdS system under different crystal growth conditions, the formation energies of the system with different doping positions are calculated as follows:

$E_{f}=E_{d}-E_{p}-m m_{S n}+m m_{C d}$

Where $E_{d}$ and $E_{p}$ represent the total energy of Sn doped CdS system and pure CdS respectively; $m_{S n}$ and $\mathrm{m}_{\mathrm{Cd}}$ represents the chemical potential of $\mathrm{Sn}$, respectively; $\mathrm{m}$ is doped atom number. The chemical potential can be obtained from the ground state energy of bulk materials $\mathrm{m}_{\mathrm{Cd}}$. The chemical potential of $\mathrm{Cd}$ and $\mathrm{S}$ in CdS structure is as follows:

$\mathrm{m}_{\mathrm{CdS}} \otimes \mathrm{m}_{\mathrm{Cd}} \rrbracket \mathrm{m}_{\mathrm{S}}$

Where $\mathrm{m}_{\mathrm{Cd}}$ and $\mathrm{m}_{\mathrm{S}}$ denote the chemical potential of pure $\mathrm{Cd}$ and $\mathrm{S}$ atoms, respectively. The populations and energies of CdS doped by $\mathrm{Sn}$ are listed in table 1. It can be seen from table 1 that with the decrease of $\mathrm{Sn}$ doping concentration, the value of the formation energy $\Delta \mathrm{E}$ from $-2.86 \mathrm{eV}$ decrease to $-21.65 \mathrm{eV}$ which means the lower the doping concentration is, the lower the formation energy is, and the higher the stability is. The population can be used to analyze the strength of covalent properties of chemical bonds. Table 1 shows that the maximum bond population of various doping concentrations have little change. From the bond population data, it can be seen that with the increase of Sn doping ion concentration, the bond population decreases steadily, which also indicates that the stability of Sn doped CdS at low doping ratio. 
Fig. 2 shows the energy band structure diagrams of the supercells of CdS of doped by Sn. Fig.2a shows the calculated band gap of pure CdS. Fermi level is set as zero, and the top of the valence band is mainly composed of S-3p orbital while the bottom of the conduction band is composed of Cd-5s and Cd-4p orbitals, and it can be seen that pure CdS is a $N$ typed semiconductor with a band gap $\left(E_{g}\right)$ of 2.45 eV by LDA+U method, which is consistent with the experimental results and more accurate than that of LDA and GGA method [29,30]. Fig.2b-e are the energy band gap diagram of Sn doped CdS. It can be seen obviously that there is an impurity band in the Fermi level, while the valence band is below it and conduction band is above it, which makes the absorption of photons have three ways: one is from the valence band directly to the conduction band, the other is from the valence band to the intermediate band, and the other is from the intermediate band to the conduction band, while the band gap remains unchanged. Refer to Fig. 3, the intermediate band is made up of the interaction of all the Sn-5p, S-3p and $\mathrm{Cd}-4 \mathrm{~s}$ orbitals as shown in Fig3b. Such results indicate that the doping of Sn can induce intermediate band structure of CdS.

Charge density difference diagrams of CdS doped by Sn are shown Fig.4, and it is an effective method to study the redistribution of electrons when materials are doped. The gain, loss and transfer of electrons around doped atoms can be clearly seen by charge density difference diagram, by which the bonding characteristics and interaction between atoms can be judged. In order to further study the mechanism of the effect of doped atoms on the electronic structure, the charge density difference diagram along the (001) crystal plane is shown in fig. 4. Due to the complexity of the relative position of doped atoms in the crystal cell, we only discuss the charge density difference changes of CdS before and after doping (Sn doping concentration is $1 / 16$ ) here. The value of the scale bar represents the value of the charge density difference contour, which reflects the bonding type, charge distribution and charge transfer between atoms. The blue area indicates the area where electrons are obtained, the red area indicates the area where electrons are lost. The darker the color, the more electrons are gained or lost. It can be seen from fig. 4 that Sn doping provides more free electrons than $\mathrm{Cd}$. The charge density difference around the Sn doped atoms increases obviously. The Sn atom is surrounded by yellow color, indicating that Sn atoms lose electrons and are donor atoms. The dark blue represents $\mathrm{S}$, indicating that $\mathrm{S}$ atoms get electrons and are acceptor atoms. The color between Sn and Cd atoms is yellow and evenly distributed, the gain and loss of surface electrons are relatively large, thus there are more free electrons in Sn doping system than that of pure CdS.

Fig. 5 shows the relationship between optical absorption coefficient and optical wavelength of CdS with different Sn doping concentrations. It can be seen that the absorption coefficient after doping significantly increases in visible region. When Sn doping concentration is $1 / 16$, the optical absorption edge redshifts and the absorption coefficient and reflectivity increase obviously in the near UV-visible region (200-780nm). When Sn doping concentration is increased to $1 / 8$ and $1 / 4$, the absorption coefficient in the visible region decreases and the reflectivity (Fig.6) decreases compared with that of CdS with Sn doping concentration of $1 / 16$. This is because the energy level curve at the top of the valence band is thinning and the electron distribution decreases when doping concentration is higher than 1/8. 
The number of electrons at the top of the valence band and the bottom of the conduction band decrease, resulting in the decrease of the photon absorption capacity. Therefore, from the results of photoabsorption coefficient and reflectivity, the optimum Sn doping ratio is 1/16.

Dielectric function is a common index to study the optical properties of materials. The macroscopic optical property of solids is generally expressed by complex dielectric functions:

$\varepsilon(\omega)=\varepsilon_{1}(\omega)+i \varepsilon_{2}(\omega)$

Where $\omega$ is the frequency, and $\varepsilon_{1}(\omega)$ and $\varepsilon_{2}(\omega)$ are the real and imaginary parts of the dielectric function, respectively. The imaginary part $\varepsilon_{2}(\omega)$, which means the power loss, is directly related to optical absorption, and the real part $\varepsilon_{1}(\omega)$ can be calculated by Kramers-Krönig relation calculation. Absorption coefficients and reflectivity can be given by $\varepsilon_{1}(\omega)$ and $\varepsilon_{2}(\omega)$ :

$$
\begin{aligned}
& \alpha(\omega)=\sqrt{2} \omega\left[\sqrt{\varepsilon_{1}{ }^{2}(\omega)+\varepsilon_{2}{ }^{2}(\omega)}\right]^{0.5} \\
& R(\omega)=\left|\frac{\sqrt{\varepsilon_{1}(\omega)+\mathrm{j} \varepsilon_{2}(\omega)}-1}{\sqrt{\varepsilon_{1}(\omega)+\mathrm{j} \varepsilon_{2}(\omega)}+1}\right|^{2}
\end{aligned}
$$

Where $a(\omega)$ and $R(\omega)$ are the absorption coefficient and reflectivity, respectively. Fig. 7 shows the curves of imaginary parts of the dielectric function of Sn doped ZnS with different doping concentration. As we can see, the imaginary part of the dielectric function in the UV-visible region increases in intensity after Sn doping. The imaginary part of the dielectric function is the largest when the Sn doping ratio to $\mathrm{Zn}$ is $1 / 16$, and it has two wide peak in visible region, indicating that the power loss is biggest, the trend of which is the same as the conductivity in fig.8. By comparison with fig. 3 and fig. 4 , it can be seen that when the doping ratio is $1 / 16$, the impurity level has an intermediate band structure, but the power loss and conductivity are both large, and the conductivity also increases sharply as shown in fig.8. At such a doping ratio, CdS doped by Sn gives rise to an excellent intermediate band structure.

\section{Conclusions}

In this paper, the band structure, state density and optical properties of CdS doped by Sn, the doping ratio of which is $1 / 32,1 / 16,1 / 8$ and $1 / 4$, are calculated by the first principles of density functional theory of plane wave pseudopotential method. The results show that CdS can produce the intermediate band structure when Sn doping, and when Sn doping ratio is $1 / 16, \mathrm{CdS}$ has the maximum light absorption coefficient and conductivity.

\section{Declarations}

\section{Acknowledgement}


This work was supported by Gansu Provincial Youth Science and Technology Fund Projects of China (Grant No.1506RJYA093).

\section{Conflict of Interest}

The authors declare that there is no conflict of interest.

\section{Data availability statements}

All data generated or analysed during this study are included in this published article.

\section{References}

[1] Yao H, Zhang C, Wang Q, et al. Novel Two-Dimensional Layered MoSi2Z4 (Z = P, As): New Promising Optoelectronic Materials[J]. Nanomaterials, 2021, 11(3):559.

[2] Li X, Xiao D, Wu L, et al. CdTe thin film solar cells with copper iodide as a back contact buffer layer[J]. Solar Energy, 2019, 185:324-332.

[3] Cui Y H, Tian L J, Li W W, et al. Solar-energy-facilitated CdSxSe1-x quantum dot bio-assembly in Escherichia coli and Tetrahymena pyriformis[J]. Journal of Materials Chemistry A, 2019, 7(11).

[4] Lee M M, Teuscher J, Miyasaka T, et al. Efficient hybrid solar cells based on meso-structured organometal halide perovskites[J]. Science, 2012, 338(6107):643-647.

[5] P Péchy, Renouard T, Zakeeruddin S M , et al. Engineering of Efficient Panchromatic Sensitizers for Nanocrystalline $\mathrm{TiO}_{2}$-Based Solar Cells[J]. Journal of the American Chemical Society, 2001, 123(8):161324.

[6] Jibran M, Sun X, J Hua, et al. $\mathrm{Cu}_{2} \mathrm{Zn}(\mathrm{Si}, \mathrm{Ge}) \mathrm{Se}_{4}$ quaternary semiconductors as potential photovoltaic materials[J]. Chemical Physics Letters, 2020, 756:137820.

[7] Vaishanav S K, Korram J, Nagwanshi R, et al. Interaction of Folic Acid with Mn Doped CdTe/ZnS Quantum Dots: In Situ Detection of Folic Acid[J]. Journal of Fluorescence, 2021:1-10.

[8] Zhang Y, Ye J, Liu Z, et al. Red-emissive poly(phenylene vinylene)-derivated semiconductors with wellbalanced ambipolar electrical transporting properties[J]. Journal of Materials Chemistry C, 2020, 8.

[9] Yu J, Deng H, Zhang Q, et al. The role of tuning Se/(S + Se) ratio in the improvement of $\mathrm{Cu}_{2} \mathrm{MnSn}(\mathrm{S}$, $\mathrm{Se})_{4}$ thin films properties and photovoltaic device performance[J]. Solar Energy, 2019, 179(FEB.):279-285. 
[10] Yu Q, Pan J, Mei J, et al. The $\mathrm{Cu}_{2} \mathrm{O} / \mathrm{CuO} / \mathrm{SnO}_{2}$ transparent pn junction film device towards photovoltaic enhancement with Cu self-oxidation transition layer[J]. Journal of Materials Science, 2021:112.

[11] Liu H, Liu H R, Yang F, et al. $\pi$-Conjugated small molecules enable efficient perovskite growth and charge-extraction for high-performance photovoltaic devices[J]. Journal of power sources, 2020, 448: 227420-227420.

[12] Zhang P, Luan D, Lou X. Fabrication of CdS Frame-in-Cage Particles for Efficient Photocatalytic Hydrogen Generation under Visible-Light Irradiation[J]. Advanced Materials, 2020, 32: 2004561

[13] Maity P, Kumar S, Kumar R, et al. Role of Cobalt Doping in CdS Quantum Dots for Potential Application in Thin Film Optoelectronic Devices[J]. The Journal of Physical Chemistry C, 2021, 125.

[14] Liu R, Hao J, Li J, et al. Causal Inference Machine Learning Leads to Original Experimental Discovery in CdSe/CdS Core/Shell Nanoparticles[J]. Journal of Physical Chemistry Letters, 2020, 17(17):7232-7238.

[15] Wan W, Zhang M, Zhao M, et al. Room-temperature formation of CdS magic-size clusters in aqueous solutions assisted by primary amines[J]. Nature Communications, 2020, 11(1):4199.

[16] AF Gualdrón-Reyes, AM Meléndez, Tirado J, et al. Hidden energy levels? Carrier transport ability of $\mathrm{CdS} / \mathrm{CdS}_{1-\mathrm{x}} \mathrm{Se}_{\mathrm{x}}$ quantum dot solar cells impacted by $\mathrm{Cd}-\mathrm{Cd}$ level formation[J]. Nanoscale, 2019, 11(2):762-774.

[17] Wang L , Zhao X, Yang Z, et al. CuSbS 2 Solar Cells Using CdS, $\ln _{2} S_{3}$ and the $\ln /$ Cd-based Hybrid Buffers[J]. Journal of Electronic Materials, 2021:1-5.

[18] Feng J, Ma X, Liao J, et al. A potential alternative deposition technology for CdS buffer layer in kesterite solar cells via intermittent photochemical deposition[J]. Applied Surface Science, 2020, 527:146911.

[19] Madhavi J, Prasad V B, Raghu A V. Synthesis of composite nanopowder through Mn doped ZnS-CdS systems and its structural, optical properties[J]. Journal of Molecular Structure, 2021, 1230(26):129875.

[20] X Zhou, Fang Y, X Cai, et al. In Situ Photodeposited Construction of Pt-CdS/g- $\mathrm{C}_{3} \mathrm{~N}_{4}-\mathrm{MnO} \times$ Composite Photocatalyst for Efficient Visible-Light-Driven Overall Water Splitting[J]. ACS Applied Materials \& Interfaces, 2020, 12(18).

[21] Li X, M Edelmannová, Huo P, et al. Fabrication of highly stable CdS $/ \mathrm{g}-\mathrm{C}_{3} \mathrm{~N}_{4}$ composite for enhanced photocatalytic degradation of $\mathrm{RhB}$ and reduction of $\mathrm{CO}_{2}[\mathrm{~J}]$. Journal of Materials Science, 2020, 55(8).

[22] Liu H, Hao J, Li J, et al. Spectral and Nonlinear Optical Properties of Quasi-Type II CdSe/CdS

Nanotadpoles[J]. The Journal of Physical Chemistry C, 2020, 124(50):27840-27847. 
[23] Tan P, Ca N, Hien N T, et al. New insights on the energy transfer mechanisms of Eu-doped CdS quantum dots[J]. Physical Chemistry Chemical Physics, 2020, 22(11).

[24] Ramiro I, A Martí. Intermediate band solar cells: Present and future[J]. Progress in Photovoltaics: Research and Applications, 2020.

[25] Xue Y, Zhou W, Ding L, et al. First-principles study on Sb-doped $\mathrm{SnS}_{2}$ as a low cost and non-toxic absorber for intermediate band solar cell[J]. Physics Letters A, 2020, 384(27):126695.

[26] WIfa B, Hyy B, Ylw A, et al. Fabrication of a Ti-substituted $\mathrm{CuGaS}_{2}$ intermediate band material by alternate sputtering [J]. Materials Letters, 2019, 236:23-25.

[27] Tanaka T, Matsuo K, Saito K, et al. Cl-doping effect in $\mathrm{ZnTe}_{(1-x)} \mathrm{O}_{\mathrm{x}}$ highly mismatched alloys for intermediate band solar cells[J]. Journal of Applied Physics, 2019, 125(24):243109-243109.

[28] Tanoh A, Gauriot N, Delport G, et al. Directed Energy Transfer from Monolayer WS 2 to Near-Infrared Emitting PbS-CdS Quantum Dots[J]. ACS nano, 14(11):15374-15384.

[29] A S O , A S G , A H M , et al. Theoretical study of structural, electronic, and thermal properties of CdS, CdSe and CdTe compounds[J]. Computational Materials Science, 2011, 50(4):1460-1466.

[30] Junaid M , Khan I, Kanwal Z, et al. Investigating structural, electronic and optical properties of CdS:Cr (A GGA and GGA+U study)[J]. Solid State Sciences, 2020, 108.

\section{Tables}

Table 1. The populations and energies of CdS doped by Sn.

\begin{tabular}{lllll}
\hline $\begin{array}{l}\text { Doping } \\
\text { models }\end{array}$ & $\begin{array}{l}\text { Cd-S } \\
(\mathbf{m a x})\end{array}$ & $\begin{array}{c}\text { Cd-S } \\
(\mathbf{m i n})\end{array}$ & $\begin{array}{l}\text { Cd-S } \\
(\mathbf{m i n} / \mathbf{m a x})\end{array}$ & $\Delta \mathbf{E} / \mathbf{e V}$ \\
\hline $\mathbf{C d}_{32} \mathbf{S}_{32}$ & 0.34 & 0.34 & $100.00 \%$ & $/$ \\
\hline $\mathbf{C d}_{31} \mathbf{S n}_{1} \mathbf{S}_{32}$ & 0.47 & 0.46 & $97.87 \%$ & $\mathbf{- 2 1 . 6 5}$ \\
\hline $\mathbf{C d}_{30} \mathbf{S n}_{2} \mathbf{S}_{32}$ & 0.44 & 0.37 & $84.09 \%$ & $\mathbf{- 1 4 . 3 6}$ \\
\hline $\mathbf{C d}_{28} \mathbf{S n}_{4} \mathbf{S}_{32}$ & 0.42 & 0.35 & $83.33 \%$ & $\mathbf{- 8 . 7 2}$ \\
\hline $\mathbf{C d}_{24} \mathbf{S n}_{8} \mathbf{S}_{32}$ & 0.40 & 0.32 & $80.00 \%$ & $\mathbf{- 2 . 8 6}$ \\
\hline
\end{tabular}

\section{Figures}



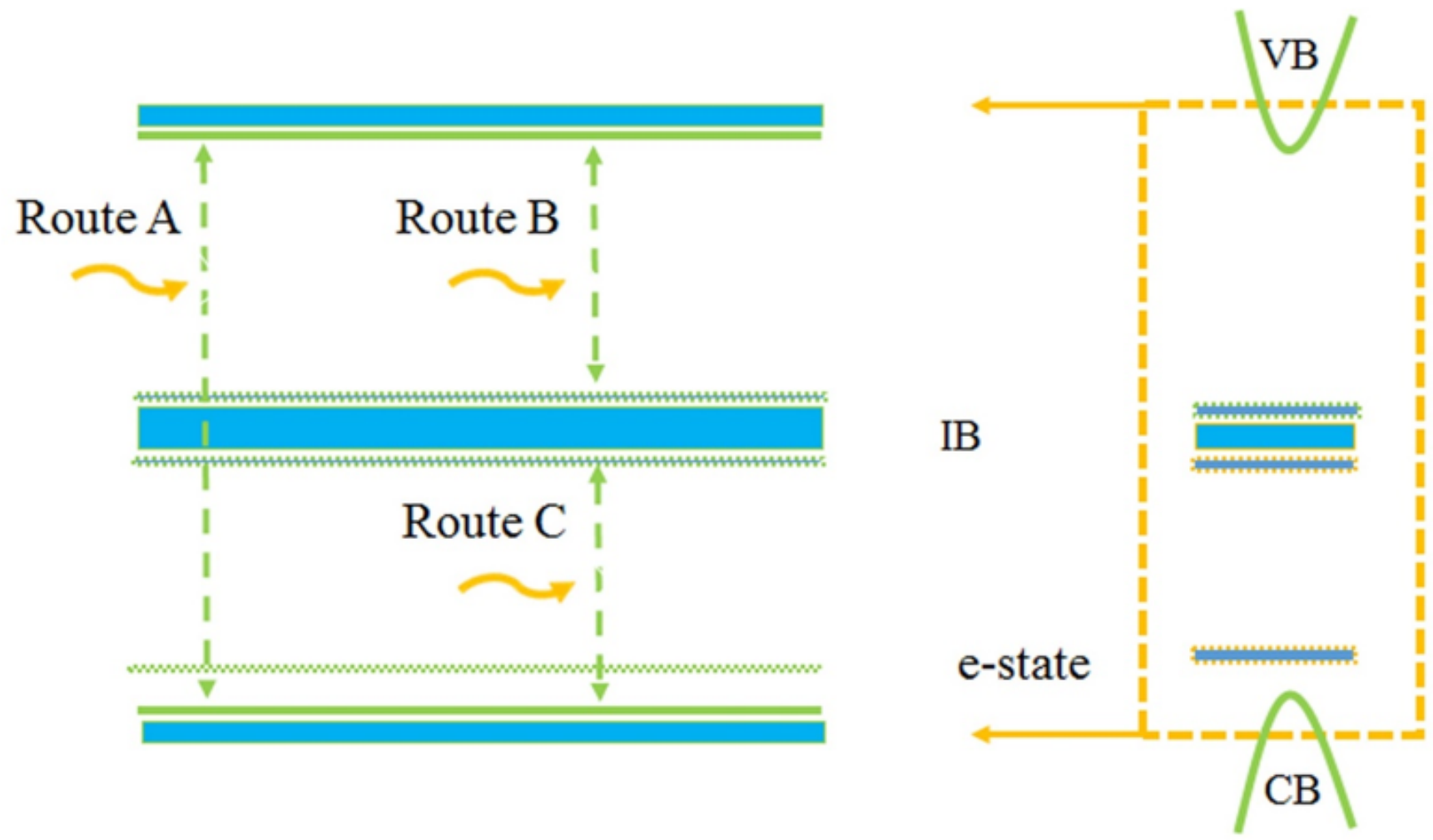

Figure 1

model of intermediate band. 

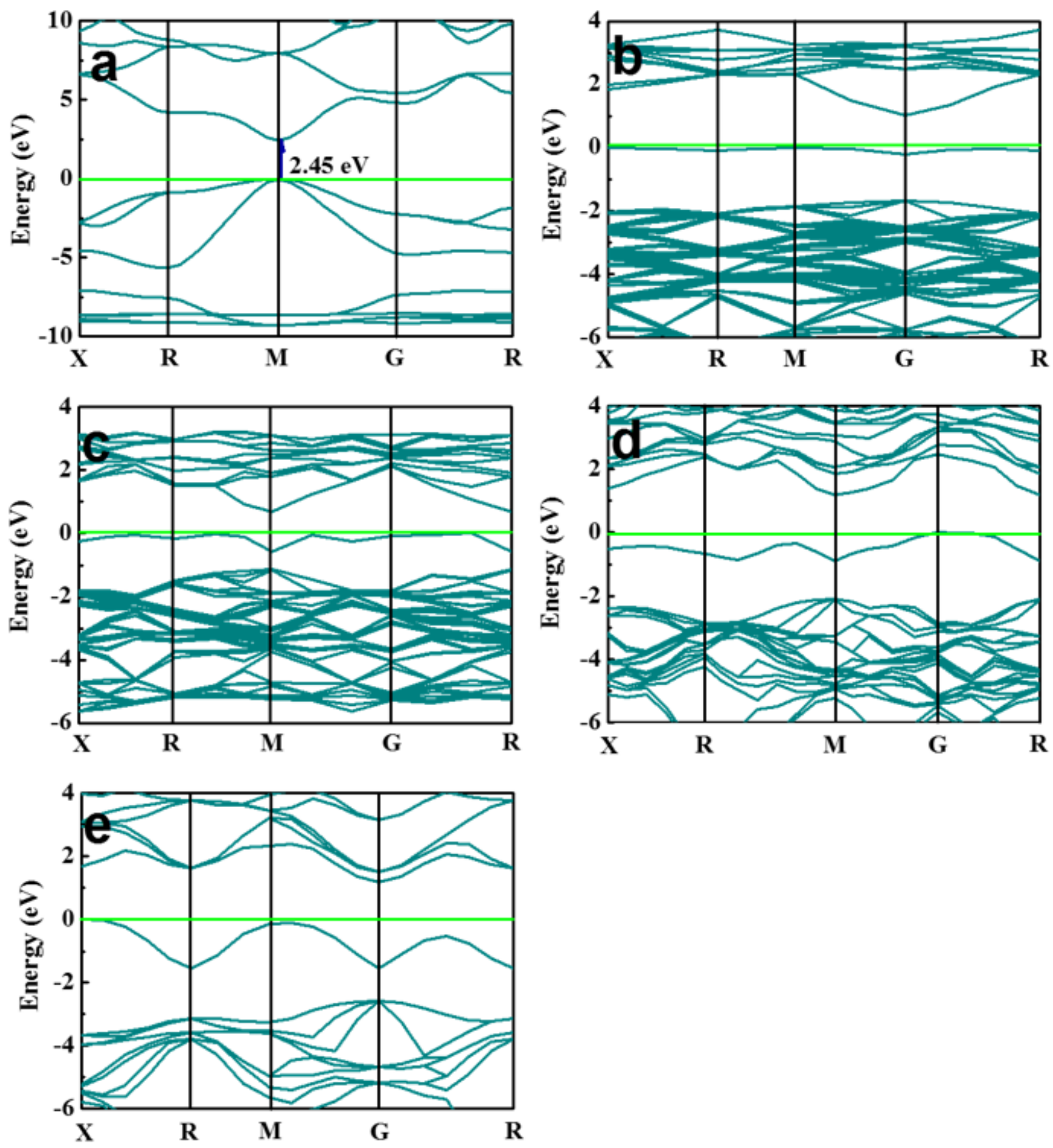

Figure 2

the band structure diagrams of Sn doped CdS. (a) the band structure of pure CdS; (b) the band structure of CdS with Sn doping concentration of 1/32; (c) the band structure of CdS with Sn doping concentration of 1/16; (d) the band structure of CdS with Sn doping concentration of 1/8; (e) the band structure of CdS with Sn doping concentration of 1/4. 


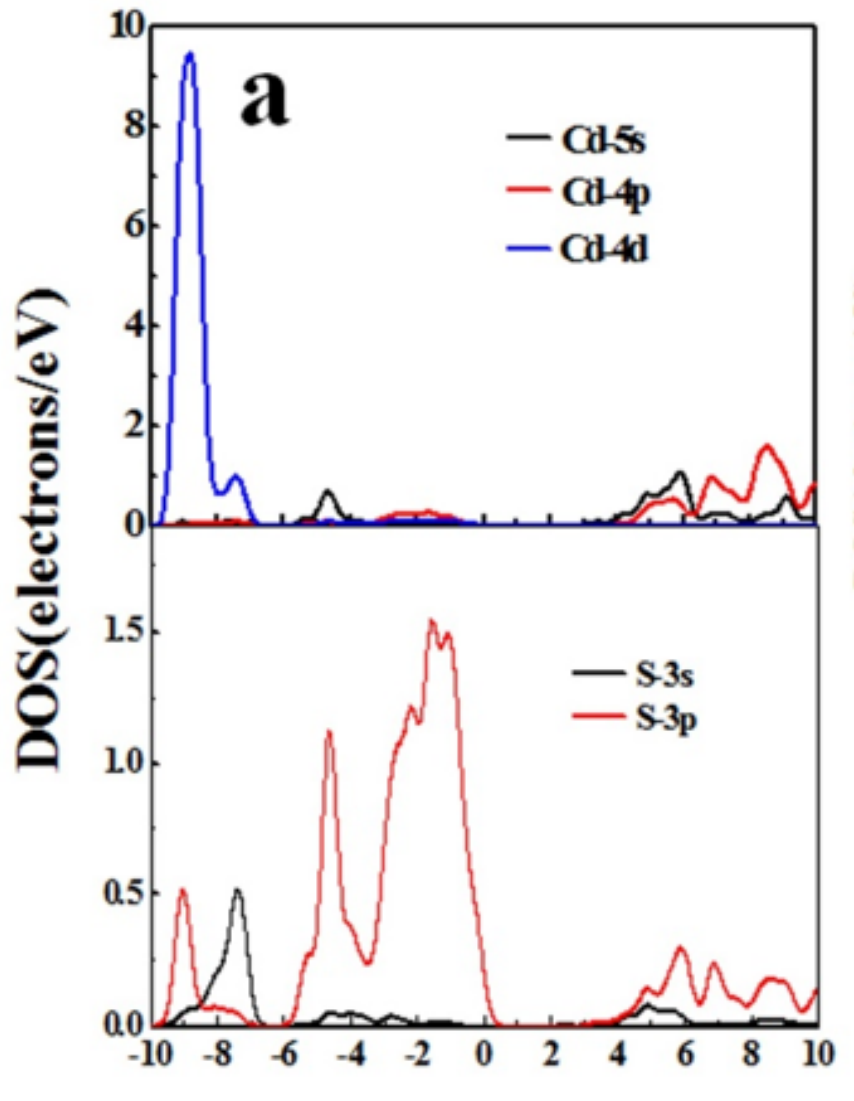

Energy (eV)

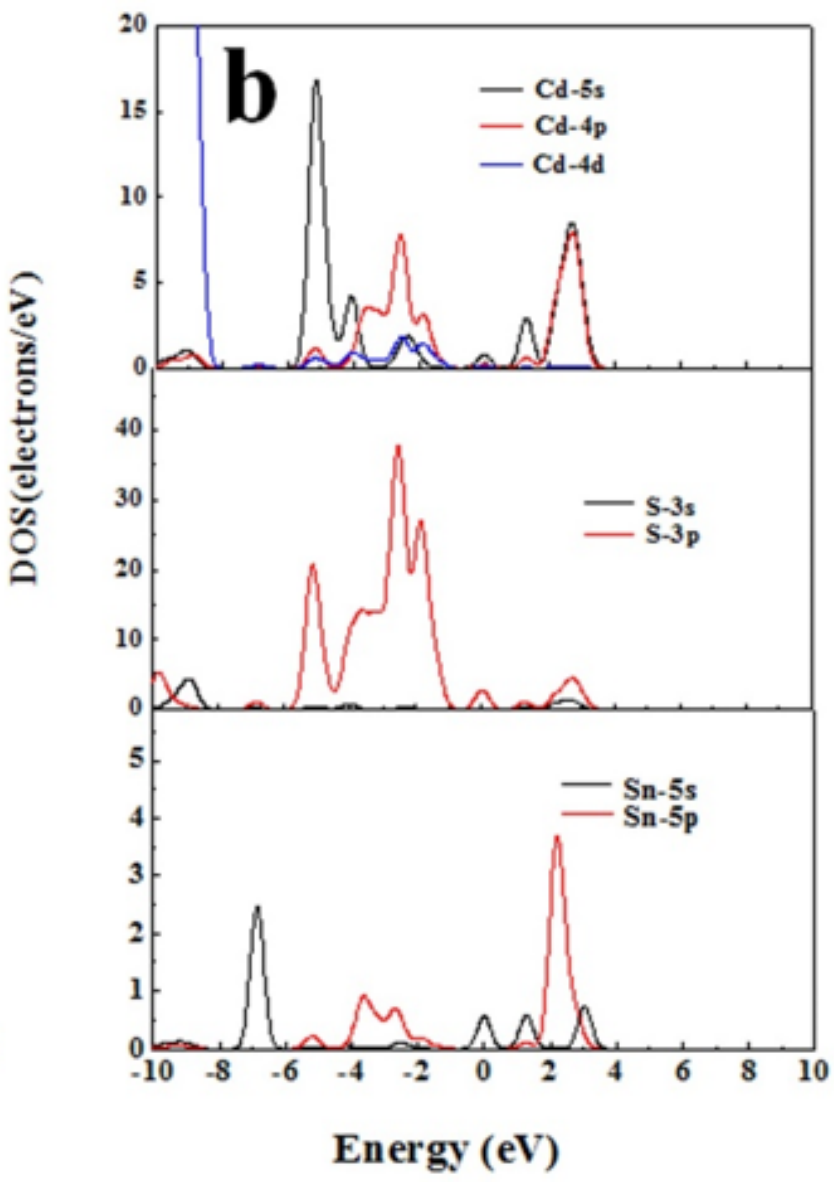

Energy (eV)

\section{Figure 3}

the density of states diagrams of Sn doped CdS. (a) the density of states diagrams of pure CdS; (b) the density of states diagrams of CdS with Sn doping concentration of 1/16. 


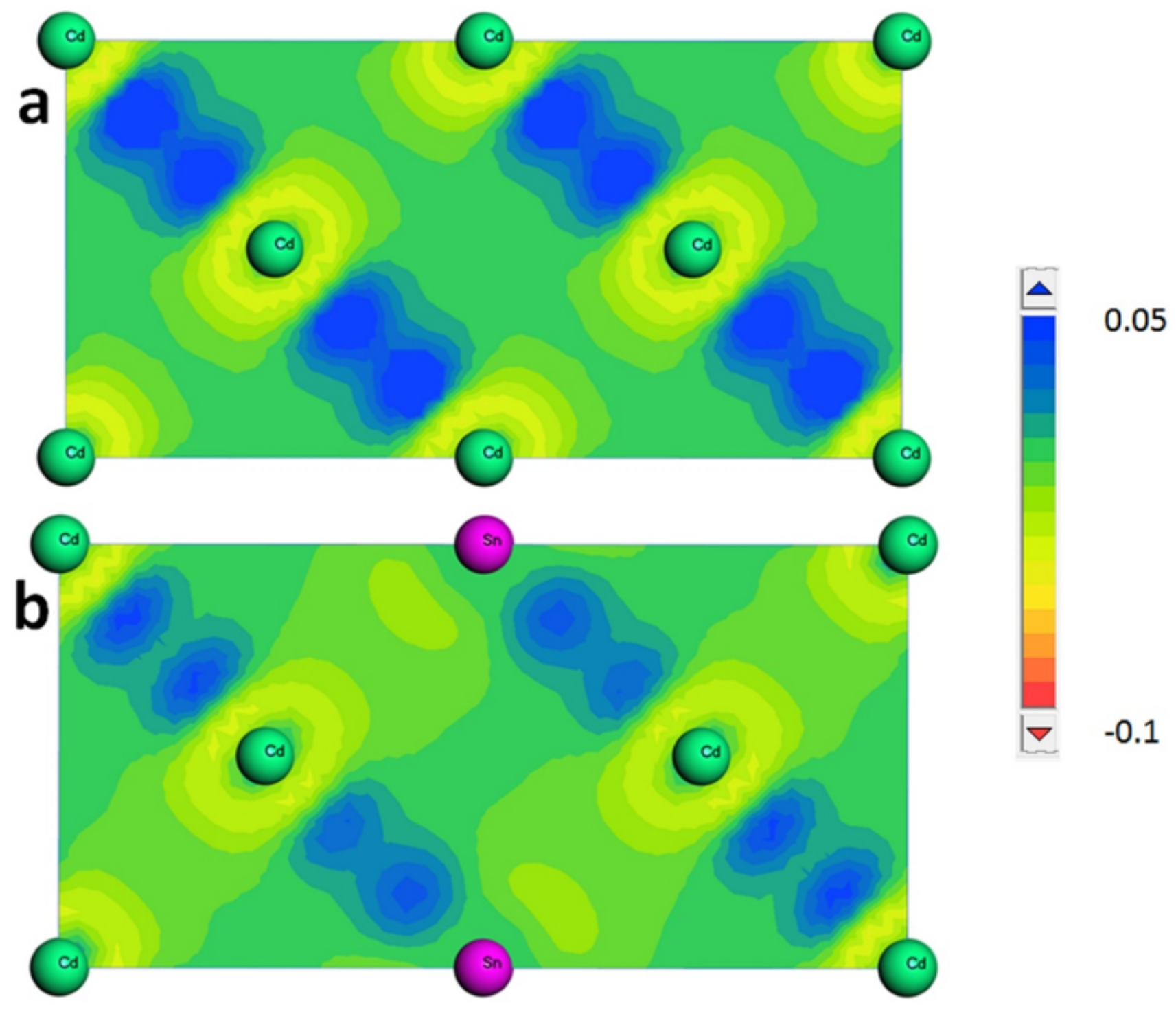

Figure 4

charge density difference of Sn doped CdS. (a) charge density difference of pure CdS; (b) charge density difference of CdS with Sn doping concentration of 1/16. 


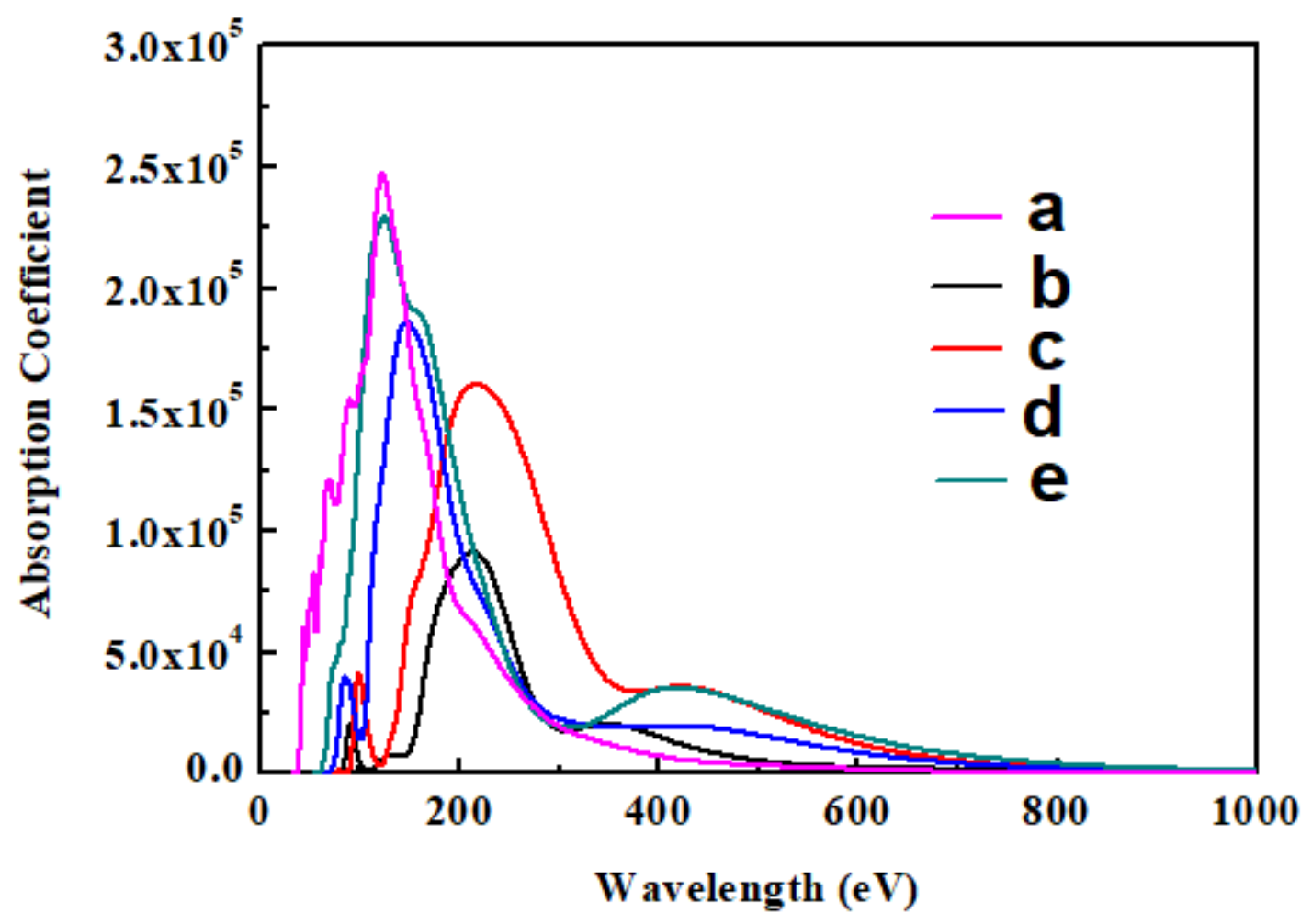

Figure 5

absorption coefficient of Sn doped CdS. (a) absorption coefficient of pure CdS; (b) absorption coefficient of CdS with Sn doping concentration of 1/32; (c) absorption coefficient of CdS with Sn doping concentration of 1/16; (d) absorption coefficient of CdS with Sn doping concentration of 1/8; (e) absorption coefficient of CdS with Sn doping concentration of 1/4. 


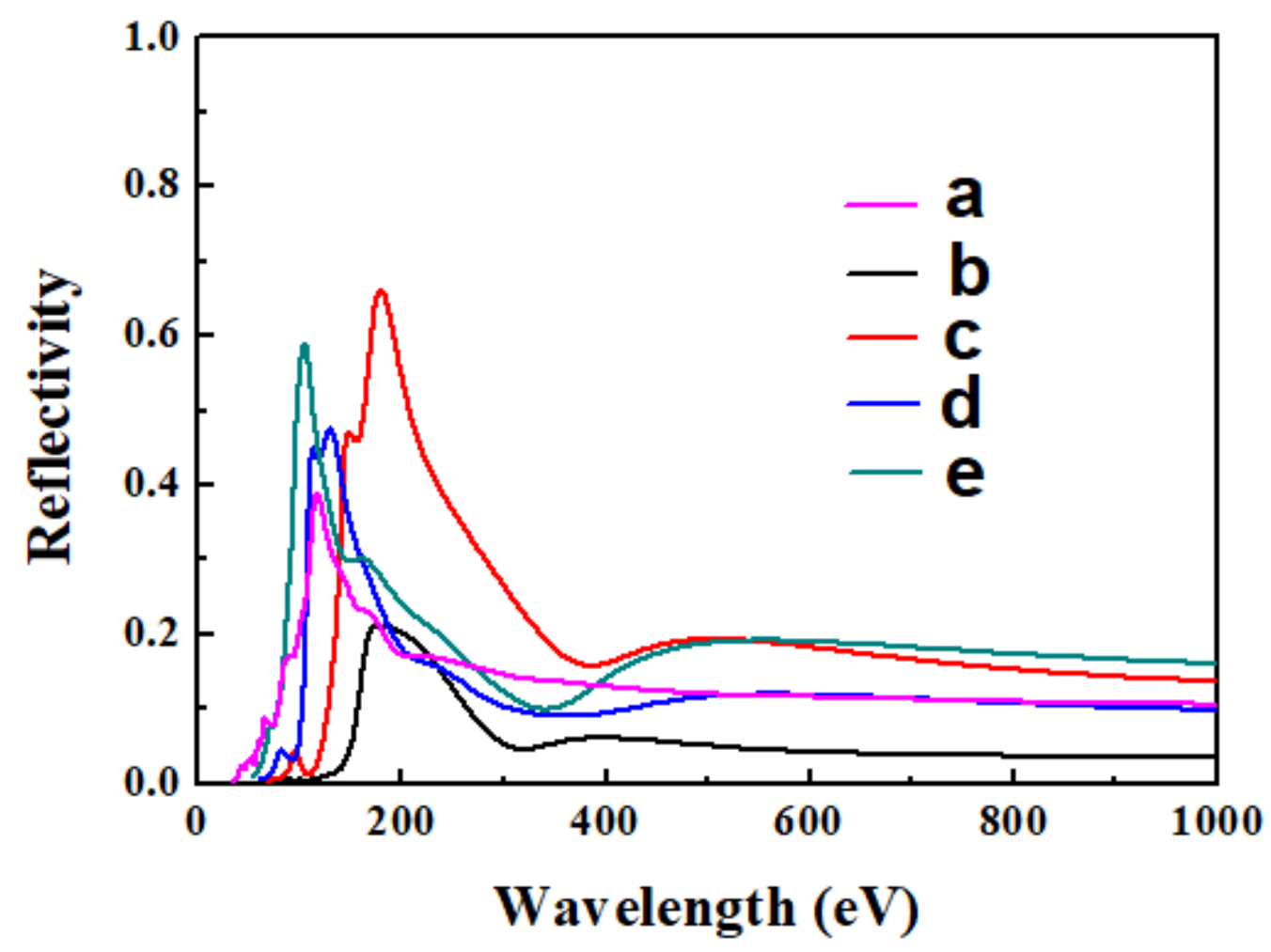

Figure 6

reflectivity of Sn doped CdS. (a) reflectivity of pure CdS; (b) reflectivity of CdS with Sn doping concentration of $1 / 32$; (c) reflectivity of CdS with Sn doping concentration of 1/16; (d) reflectivity of CdS with Sn doping concentration of 1/8; (e) reflectivity of CdS with Sn doping concentration of 1/4. 


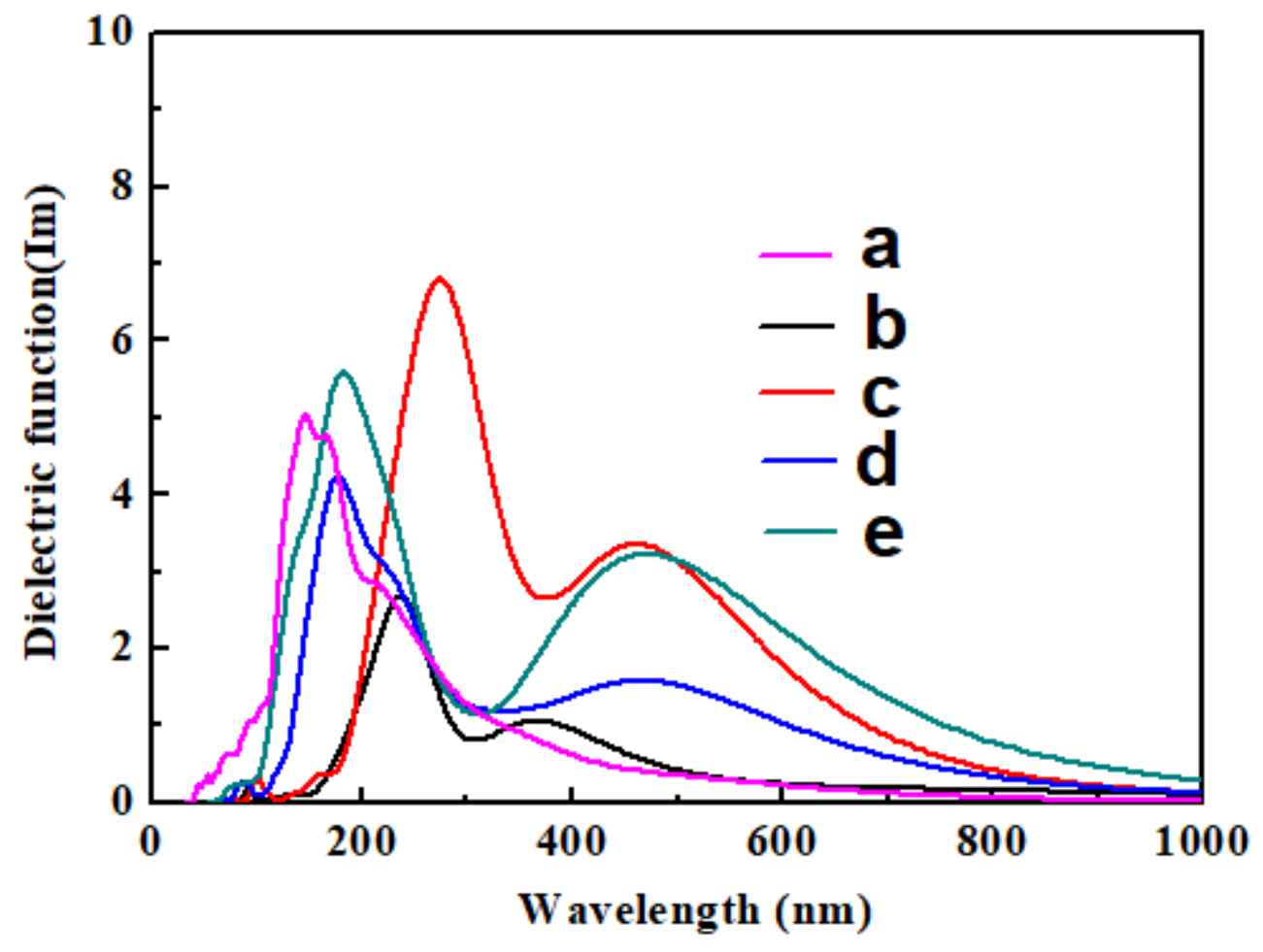

Figure 7

imaginary part of dielectric function of Sn doped CdS. (a) imaginary part of dielectric function of pure CdS; (b) imaginary part of dielectric function of CdS with Sn doping concentration of 1/32; (c) imaginary part of dielectric function of CdS with Sn doping concentration of 1/16; (d) imaginary part of dielectric function of CdS with Sn doping concentration of 1/8; (e) imaginary part of dielectric function of CdS with Sn doping concentration of $1 / 4$. 


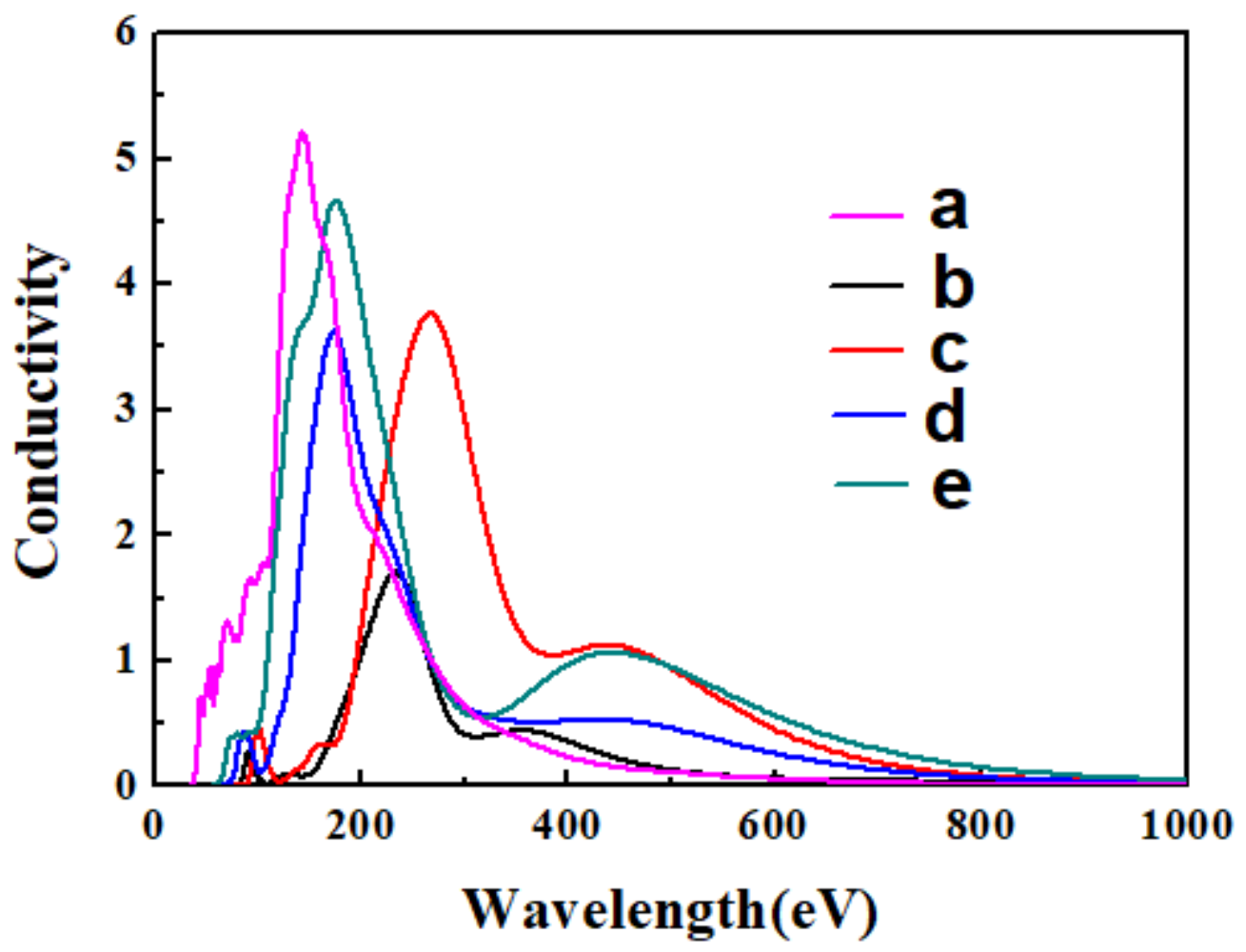

Figure 8

conductivity of Sn doped CdS. (a) conductivity of pure CdS; (b) conductivity of CdS with Sn doping concentration of 1/32; (c) conductivity of CdS with Sn doping concentration of 1/16; (d) conductivity of CdS with Sn doping concentration of 1/8; (e) conductivity of CdS with Sn doping concentration of 1/4. 